\title{
Therapie der aktinischen Keratose
} Wer nur einzelne Läsionen hat und Schmerz verträgt, für den kann die Behandlung in einigen
Sekunden vorüber sein. Andere cremen monatelang, dafür aber mit besten kosmetischen Ergebnis-
sen. Wir sprachen mit Dr. Peter Mohr über die Behandlungsmöglichkeiten bei aktinischer Keratose.

?Wovon hängt die Wahl der Therapie bei der aktinischen Keratose ab? Mohr: Es gibt keinen in einer Leitlinie verabschiedeten Therapiealgorithmus, vielmehr muss sich der Arzt individuell auf den jeweiligen Patienten einstellen. Einzelläsionen kann man vereisen, mit Laser oder Elektrokauter behandeln oder man kann eine Kürettage durchführen. Sind größere Flächen betroffen, sollte ein Therapiekonzept entwickelt werden. Die Wahl der Methode hängt vor allem davon ab, wie viel Zeit der Patient investieren will, wie viel Schmerz er erträgt und inwieweit er die Nebenwirkungen toleriert.

Viele Dermatologen beginnen die Behandlung mit einer Creme mit den Wirkstoffen Hyaluronsäure und Diclofenac. Diese muss über drei Monate zweimal täglich aufgetragen werden. Wirkt sie nicht, kann man zur nächsten Stufe übergehen, etwa einer lokalen Chemotherapie oder einer immunologischen Therapie. Bleiben bei fortgeschrittenen Stadien dennoch aktinische Keratosen zurück, müssen diese mit lokalen Maßnahmen wie Vereisung oder Laser nachbearbeitet werden.

? Welchen Patienten empfehlen Sie von vorneherein eine „invasivere" Methode?

Mohr: Wenn der Patient keinen sehr hohen kosmetischen Anspruch hat oder wenn Hautregionen betroffen sind, die kosmetisch nicht sehr anspruchsvoll sind, wird man einzelne Läsionen häufig per Kryotherapie, Laser oder Kürettage entfer-

*PDT = photodynamische Therapie

\begin{abstract}
Aktinische Keratosen treten an sonnenexponierter Haut auf und sind als frühe In-situ-Karzinome vom Plattenepitheltyp anzusehen. Ihr Progressionsrisiko wird mit bis zu $16 \%$ pro Jahr angegeben. $65 \%$ der primären Plattenepithelkarzinome und $36 \%$ der Basalzellkarzinome entwickeln sich in Läsionen, die zuvor als aktinische Keratosen eingestuft worden sind. Die Prävalenz dieser neoplastischen Hautveränderungen ist mit $6-26 \%$ hoch. In Australien, dem Land mit der weltweit höchsten Hautkrebsrate, weisen $40-60 \%$ der Erwachsenen aktinische Keratosen auf.
\end{abstract}

Aktinische Keratosen nicht unterschätzen

Dr. Robert Bublak

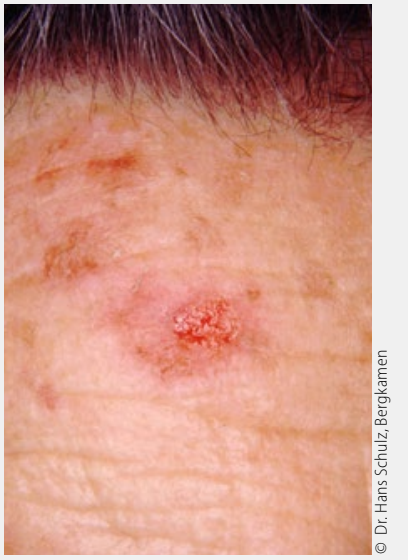

Plattenephithelkarzinom auf aktinischer Keratose.

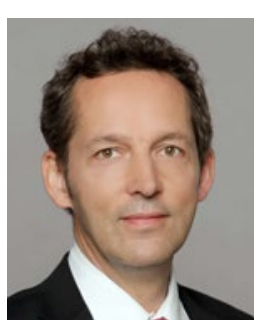

Dr. med. Peter Mohr

Chefarzt der Klinik für Dermatologie

Hautkrebszentrum Buxtehude, Elbe Kliniken

Das Einzige, was präventiv etwas bringt, ist Lichtschutz.

nen. Das Ganze ist mit einer einzigen Behandlung oft erledigt und heilt in zwei bis längstens vier Wochen ab. Ob diese Methoden zum Einsatz kommen, hängt allerdings davon ab, ob der Patient bereit ist, kurzfristig den Schmerz zu ertragen und ob er ein kosmetisches Ergebnis akzeptiert, bei dem meist eine dauerhafte Hypopigmentierung entsteht.

? Wie lange dauert es, bis die Hautreizungen nach einer Behandlung mit Cremes wieder abklingen?

Mohr: Alle Cremes und Gele bis auf Hyaluronsäure-Präparate verursachen oft kräftige Nebenwirkungen, sodass man durch die auftretenden Entzündungsreaktionen über zwei bis vier Wochen etwas gezeichnet aussieht. Auch nach einer PDT* erkennt man über etwa zwei bis vier Wochen deutlich, dass eine Behandlung stattgefunden hat. Bei der PDT kommen häufig Schmerzen dazu, die extrem stark sein können. Auf der anderen Seite liefern Cremes und PDT die besten kosmetischen Ergebnisse und die PDT kann an größeren Arealen appliziert werden.

? Wie hoch ist die Wahrscheinlichkeit für ein erneutes Auftreten aktinischer Keratosen und wie kann es verhindert werden?

Mohr: Ich würde sagen $100 \%$. Denn meist liegt ja auch eine Schädigung der umgebenden Haut vor. Spätestens nach einem Jahr kommt der Betroffene mit neuen Läsionen, manchmal auch schon nach zwei Monaten. Hat jemand bereits eine Feldkanzeration, kommen die aktinischen Keratosen mit Sicherheit wieder und müssen erneut behandelt werden. Das Einzige, was präventiv etwas bringt, ist Lichtschutz. Bei Patienten, die dies sehr konsequent machen, muss ggf. Vitamin D substituiert werden.

Das Interview führte Dr. Christine Starostzik. 